\title{
Qualidade de vida relacionada à saúde de mulheres que referiram incontinência urinária no pós-parto
}

\author{
Health-related quality of life of women who reported postpartum urinary incontinence \\ Calidad de vida relacionada con la salud de las mujeres que mencionan incontinencia \\ urinaria en el posparto
}

Daniela Biguetti Martins Lopes ${ }^{1 *}$, Neide de Souza Praça².

\section{RESUMO}

Objetivo: avaliar a qualidade de vida relacionada à saúde das mulheres que referiram incontinência urinária após o parto, atendidas na Maternidade Municipal de Londrina, Paraná, Brasil. Método: estudo de coorte, realizado com 358 puérperas atendidas na maternidade campo do estudo, no período de dezembro de 2011 a setembro de 2012, a coleta de dados foi realizada em três etapas, sendo a primeira nas 48horas após o parto, a segunda no terceiro mês após o parto e a terceira no sexto mês. Os dados foram tratados estatisticamente e para avaliar a qualidade de vida relacionada à saúde das mulheres incontinentes foi utilizado o King's Health Questionnarie. Resultados: A amostra, apresentou média de idade de 24,6 anos e incidência de incontinência urinária no pós-parto de 10,5\% e 12,3\% no terceiro e sexto mês após o parto, respectivamente. A qualidade de vida relacionada à saúde, os resultados mostraram maior comprometimento nos domínios percepção geral de saúde, medidas de gravidade, impacto da incontinência, limitações das atividades diárias e limitações físicas. Conclusão: Conclui-se que a incidência de incontinência urinária é expressiva no terceiro e no sexto mês após o parto, e que a incontinência urinária afeta a qualidade de vida relacionada à saúde de mulheres incontinentes.

Palavras-chave: Saúde da Mulher, Incontinência Urinária, Pós-Parto, Enfermagem, Qualidade de Vida.

\begin{abstract}
Objective: to evaluate the health-related quality of life of women who reported post-partum urinary incontinence at the Maternidade Municipal de Londrina, Paraná, Brazil. Method: a cohort study, carried out with 358 postpartum mothers assisted in the maternity field of the study, from December 2011 to September 2012, data collection was performed in three stages, the first in 48 hours after delivery, the second stage in the third month after delivery and the third in the sixth month. The data were statistically treated and the King's Health Questionnarie was used to evaluate the health-related quality of life of incontinent women. Results: The sample had a mean age of 24.6 years and incidence of post-partum urinary incontinence of $10.5 \%$ and $12.3 \%$ in the third and sixth month after delivery, respectively. The health-related quality of life, the results showed greater impairment in the general health perception, severity measures, impact of incontinence, limitations of daily activities and physical limitations. Conclusion: It is concluded that the incidence of urinary incontinence is significant in the third and the sixth month after childbirth, and that urinary incontinence affects the quality of life related to the health of incontinent women.
\end{abstract}

Key words: Women's Health, Urinary Incontinence, Postpartum, Nursing, Quality of Life.

${ }^{1}$ Enfermeira Obstétrica, Doutora, Professor Adjunto do Departamento de Enfermagem da Universidade Estadual de Londrina (PR), Brasil. *E-mail: daniela.biguetti@gmail.com

${ }^{2}$ Enfermeira Obstétrica, Livre Docente, Professora Associada (aposentada) do Departamento de Enfermagem Materno-Infantil e Psiquiátrica da Escola de Enfermagem da Universidade de São Paulo (SP), Brasil. 


\section{RESUMEN}

Objetivo: evaluar la calidad de vida relacionada a la salud de las mujeres que mencionaron incontinencia urinaria después del parto, atendidas en la Maternidad Municipal de Londrina, Paraná, Brasil. Metodo: Se trata de un estudio de cohorte, realizado con 358 puérperas atendidas en la maternidad campo del estudio, en el período de diciembre de 2011 a septiembre de 2012, la recolección de datos fue realizada en tres etapas, siendo la primera en las 48 horas después del parto, la segunda etapa en el tercer mes después del parto y la tercera en el sexto mes. Los datos fueron tratados estadísticamente y para evaluar la calidad de vida relacionada con la salud de las mujeres incontinentes se utilizó el King's Health Questionnarie. Resultados: La muestra, presentó una media de edad de 24,6 años e incidencia de incontinencia urinaria en el posparto del $10,5 \%$ y el $12,3 \%$ en el tercer y sexto mes después del parto, respectivamente. A la calidad de vida relacionada con la salud, los resultados mostraron un mayor compromiso en los ámbitos de percepción general de salud, medidas de gravedad, impacto de la incontinencia, limitaciones de las actividades diarias y limitaciones físicas. Conclusión: Se concluye que la incidencia de incontinencia urinaria es expresiva en el tercer y sexto mes después del parto, y que la incontinencia urinaria afecta la calidad de vida relacionada con la salud de mujeres incontinentes.

Palabras clave: Salud de la Mujer, Incontinencia Urinaria, Post-Parto, Enfermería, Calidad de Vida.

\section{INTRODUÇÃO}

A incontinência urinária (IU), definida como qualquer queixa de perda involuntária de urina (ABRAMS P, et al., 2005), pode causar impacto negativo na qualidade de vida relacionada a saúde (QVRS) em diferentes fases da vida e, sua prevalência e incidência no pós-parto podem variar de acordo com a população, o período estudado e a metodologia utilizada nas pesquisas (FARIA CA, et al., 2015, LASSERE A, et al., 2009 e MENEZES GMD, et al., 2012).

Desse fato decorre a necessidade de se investigar a IU no pós-parto, como uma das intercorrências neste período, e também, analisar a QVRS da mulher incontinente para favorecer a busca por maior compreensão da sua condição pela equipe interdisciplinar, de modo a favorecer a definição de alternativas concretas para melhor assisti-la (RAZA-KHAN F, et al., 2006). Estes achados reforçam a relevância das investigações sobre a ocorrência de IU assim como pesquisar sobre a QVRS da mulher que apresenta esta morbidade.

A qualidade de vida (QV) é definida como um conceito amplo, que incorpora, de forma complexa, a saúde física, o estado psicológico, o nível de independência, as relações sociais, as crenças pessoais e a relação com aspectos significativos do meio ambiente (WHOQOL, 1995). Refere-se, ainda, ao impacto negativo na QV, por causar repercussões na saúde sexual, psicológica e social da mulher (FARIA CA, et al., 2015, WHOQOL, 1995 e RIESCO MLG, et al., 2014).

O interesse em avaliar a QVRS das mulheres incontinentes surgiu a partir dos resultados encontrados em pesquisa anterior, realizada na cidade de São Paulo, em 2009, cujos resultados mostraram que $28,2 \%$ (2071) das mulheres incontinentes entrevistadas informaram que a perda urinária interferia na sua vida diária, de modo a afetar a QVRS. Relataram, também, que a perda de urina era motivo de constrangimento e exigia uso constante de absorvente higiênico, além de ser desconfortável, e interferir no bem-estar psicológico (LOPES DBM e PRAÇA NS, 2010).

Some-se a essa descoberta a representatividade da ocorrência de IU no pós-parto (PP), verificada na literatura, assim como sua condição de afecção clínica sub-diagnosticada. Ressalte-se o inexpressivo número de pesquisas com enfoque na prevalência, na incidência e nos fatores de risco da IU e seu impacto na QV, no país. Diante do exposto, este estudo objetivou investigar a incidência de IU no pós-parto, e avaliar a QVRS das mulheres incontinentes no terceiro e sexto mês após o parto. 


\section{MÉTODO}

Trata-se de um estudo de coorte, realizado em uma maternidade pública, localizada na região central do município de Londrina (PR). Esta maternidade é referência à gestante de risco habitual e intermediário, submetida ou não ao pré-natal na rede básica de saúde.

A população foi constituída por 358 mulheres atendidas no alojamento conjunto da Maternidade, no período de dezembro de 2011 a setembro de 2012, e que atenderam os critérios de inclusão. Por se tratar de um estudo de coorte, a coleta de dados foi realizada em três etapas: Primeira etapa foi realizada na maternidade campo de estudo nas primeiras 48 horas após o parto; segunda e terceira etapa ocorreram no terceiro e sexto mês após o parto, respectivamente. Na primeira etapa os dados foram coletados dados de identificação, clínicos e obstétricos pela pesquisadora e por outra enfermeira previamente treinada. Cabe destacar que os dados de identificação e clínicos foram informados pela entrevistada, enquanto os dados obstétricos foram obtidos de seu prontuário médico e do registro da carteira de gestante. Na segunda e terceira etapa, foram coletados dados sobre consulta puerperal, complicações no pós-parto e perda involuntária de urina, por contato telefônico, realizado pela pesquisadora.

As entrevistas foram realizadas após a concordância e a obtenção do Termo de Consentimento Livre e Esclarecido da mulher contatada na unidade de saúde campo do estudo. Para compor a coorte, as mulheres deveriam atender os seguintes critérios: ter tido parto (fator de exposição) na Maternidade campo de estudo; residir na região urbana do município de Londrina-PR; ter consentimento do responsável para as participantes menores de 18 anos; não apresentar doenças crônicas; não ter se submetido a cirurgias ginecológicas anteriores ao parto; não ter diagnóstico e/ou referir IU durante a última gestação ou em algum momento anterior, e dispor de telefone.

As entrevistadas foram classificadas em dois grupos, autorreferidas incontinentes e autorreferidas continentes, de acordo com as respostas para a questão número cinco do instrumento: "Depois do parto, a Sra. teve perda de urina?".

Os dados coletados foram codificados e armazenados, em dupla entrada, em banco de dados elaborado no software Epi-Info versão 2000 e, em seguida, foi feita a importação para o aplicativo Excel. O tratamento e análise dos dados foram feitos pelo programa $R$. Foi assumido um erro de $5 \%$ com probabilidade de $95 \%$ de certeza deste erro.

A QVRS das mulheres que referiram perda urinária no pós-parto foi avaliada com a emprego do questionário King's Health Questionnarie - KHQ. Este instrumento é recomendado pela Sociedade Internacional de Continência, por ser considerado um questionário completo que avalia tanto o impacto da IU nos diferentes domínios da QV, como os sintomas percebidos pelas pacientes.

O KHQ foi construído com foco na IU (KELLEHER CJ, et al., 1997), e validado na versão para o português (TAMANINI JTN, et al., 2003). É composto por questões agrupadas em oito domínios: percepção geral de saúde, impacto da IU, limitações de atividades diárias, limitações físicas e sociais, relacionamento pessoal, emoções, sono e disposição. Além destes domínios, são utilizadas duas escalas independentes do tipo likert, para avaliar a gravidade da IU e outra a presença e a intensidade dos sintomas urinários. Estas escalas, são graduadas em quatro opções de respostas ("nem um pouco, um pouco, moderadamente, muito" ou "nunca, às vezes, frequentemente, o tempo todo"), exceção feita ao domínio percepção geral de saúde com cinco opções de respostas ("muito boa, boa, regular, ruim, muito ruim") e ao domínio relações pessoais ("não afeta, um pouco, moderadamente e muito"). O KHQ não apresente um escore geral, cada domínio do é pontuado individualmente (TAMANINI JTN, et al., 2003).

Conforme cada domínio, a avaliação é feita de forma quantitativa (por meio de escala do tipo likert de 0 a 3 , de 1 a 4 e de 1 a 5) ou qualitativa (de nunca ou nada a muito, de não afeta a afeta muito). Esses escores são uma soma simples, transformados em uma variação de 0 a 100, e quanto maior a pontuação obtida, pior é a QVRS àquele domínio (KELLEHER CJ, et al., 1997 e TAMANINI JTN, et al., 2003). 
A confiabilidade do instrumento de avaliação da QVRS, foi avaliada pelo coeficiente Alfa de Cronbach, índice utilizado para medir a confiabilidade do tipo consistência interna de uma escala, ou seja, para avaliar a magnitude em que os itens de um instrumento estão correlacionados. Seu valor varia entre zero e um. Quanto maior for o valor, maior a consistência interna do instrumento. O valor mínimo aceitável é 0,70; abaixo desse valor a consistência interna da escala utilizada é considerada baixa (STREINER DR, 2003).

Em observância às determinações da Resolução 196/96, do Conselho Nacional de Saúde, o projeto de pesquisa foi aprovado pelo Comitê de Ética em Pesquisa da Escola de Enfermagem da Universidade de São Paulo - CEP-EEUSP, conforme o Parecer do processo no 986/2010, e autorizado pela Autarquia Municipal de Saúde da Prefeitura de Londrina (PR) conforme o documento C.D.026/2011-GES/DGTES/MAS/PML.

\section{RESULTADOS}

Os dados serão apresentados na seguinte sequência: caracterização sociodemográfica, incidência de IU autorreferida; teste de confiabilidade do instrumento KHQ; e QVRS.

\section{Características sociodemográficas}

Esta pesquisa de coorte foi constituída por 358 mulheres no período pós-parto, atendidas na maternidade campo do estudo. As mulheres entrevistadas tinham média de idade de 24,6 anos (variando de 18 a 42 anos). A maioria referiu cor da pele branca (45,2\%), 32,4\% relataram ter ensino médio completo, $88,5 \%$ viviam com companheiro e $57,8 \%$ não tinham vínculo empregatício (Tabela 1).

Tabela 1 - Características sociodemográficas das puérperas atendidas na maternidade. Londrina, 2012.

\begin{tabular}{|c|c|c|}
\hline Variável/Categoria & $\mathbf{N}$ & $\%$ \\
\hline \multicolumn{3}{|l|}{ Idade (em anos) } \\
\hline$<20$ & 83 & 23,2 \\
\hline $20-25$ & 135 & 37,7 \\
\hline $26-30$ & 74 & 20,7 \\
\hline$>30$ & 66 & 18,4 \\
\hline \multicolumn{3}{|l|}{ Cor da pele } \\
\hline Branca & 162 & 45,2 \\
\hline Negra & 40 & 11,2 \\
\hline Parda & 145 & 40,5 \\
\hline Amarela & 11 & 3,1 \\
\hline \multicolumn{3}{|l|}{ Escolaridade } \\
\hline Ensino Fundamental Incompleto & 93 & 26,0 \\
\hline Ensino Fundamental Completo & 67 & 18,7 \\
\hline Ensino Médio Incompleto & 64 & 17,9 \\
\hline Ensino Médio Completo & 116 & 32,4 \\
\hline Superior Incompleto & 8 & 2,2 \\
\hline Superior Completo & 10 & 2,8 \\
\hline \multicolumn{3}{|l|}{ Situação Marital } \\
\hline Com Companheiro & 317 & 88,5 \\
\hline Sem Companheiro & 41 & 11,5 \\
\hline Total & 358 & 100 \\
\hline
\end{tabular}

Fonte: Dados da pesquisa, 2012. 


\section{Incidência de IU autorreferida}

No terceiro mês PP (Etapa 2), a incidência autorreferida de IU na população estudada, foi de 10,5\% (35), enquanto $60 \%$ das mulheres referissem IU no primeiro mês PP. Quando a entrevista ocorreu no sexto mês PP (Etapa 3), a incidência de IU autorreferida obtida foi de 12,3\% (40); 35\% autorreferiram perdas no quinto mês PP, e nenhuma citou IU no sexto mês. Cabe ressaltar que das 40 mulheres que referiram IU no sexto mês PP, 14 (35\%) já haviam referido a ocorrência desta morbidade no terceiro mês (Tabela 2).

Tabela 2 - Período em que as mulheres identificaram a perda involuntária de urina no pós-parto. Londrina, 2012.

\begin{tabular}{|c|c|c|c|c|}
\hline \multirow[t]{2}{*}{ Início da perda de urina } & \multicolumn{2}{|c|}{ Etapa $2(n=35)$} & \multicolumn{2}{|c|}{ Etapa $3(n=40)$} \\
\hline & $N$ & $\%$ & $N$ & $\%$ \\
\hline $1^{0}$ mês PP & 21 & 60,0 & 06 & 15,0 \\
\hline $2^{\circ}$ mês PP & 10 & 28,6 & 06 & 15,0 \\
\hline 3ํmês PP & 04 & 11,4 & 02 & 5,0 \\
\hline $4^{\circ}$ mês PP & -- & -- & 12 & 30,0 \\
\hline $5^{\circ}$ mês PP & -- & -- & 14 & 35,0 \\
\hline $6^{\circ}$ mês PP & -- & -- & -- & -- \\
\hline
\end{tabular}

Fonte: Dados da pesquisa, 2012.

\section{Teste de confiabilidade do instrumento KHQ}

O coeficiente Alfa de Cronbach aplicado ao KHQ para testar sua confiabilidade junto à população do estudo encontram-se nas Tabela 3 e 4, onde se verifica que o instrumento KHQ apresentou bom nível de confiabilidade, demonstrando consistência interna satisfatória para todos os domínios. No terceiro mês (Tabela 3) de PP, o coeficiente Alfa de Cronbach padronizado geral foi de 0,93, considerado excelente, com variações de 0,55 - regular - (sono/disposição) a 0,98 - excelente (limitações das atividades diárias). Ainda que o Alfa de Cronbach, no domínio sono/disposição, apresentasse valor menor comparado aos outros domínios, não houve modificação no valor do Alfa de Cronbach geral padronizado, quando da retirada deste domínio, aumentando o seu valor para 0,94, o que indica excelente nível de consistência interna. No sexto mês (Tabela 4) o coeficiente Alfa de Cronbach padronizado geral foi de 0,96 , igualmente excelente, com variações de 0,77 - bom (medidas de gravidade) a 0,99 - excelente (limitações das atividades diárias).

Tabela 3 - Análise de confiabilidade, escores médios, desvio-padrão, mediana, valores mínimos e máximos, para os domínios do KHQ aplicado às mulheres autorreferidas incontinentes - Etapa 2 ( $n=35)$. Londrina, 2012.

\begin{tabular}{lcccccc}
\hline \multicolumn{1}{c}{ Domínios do KHQ } & $\begin{array}{c}\text { Alfa de } \\
\text { Cronbach }\end{array}$ & Média & $\begin{array}{c}\text { Desvio } \\
\text { Padrão }\end{array}$ & $\begin{array}{c}\text { Mínimo- } \\
\text { Máximo }\end{array}$ & Mediana & $\begin{array}{c}\text { IQR } \\
\text { (q3-q1) }\end{array}$ \\
\hline Percepção geral da Saúde & -- & 35,7 & 23,7 & $0,0-100,0$ & 25,0 & 25,0 \\
\hline Impacto da Incontinência & -- & 25,7 & 32,4 & $0,0-100,0$ & 33,3 & 33,3 \\
\hline $\begin{array}{l}\text { Limitações de Atividades } \\
\text { Diárias }\end{array}$ & 0,98 & 22,9 & 29,7 & $0,0-100,0$ & 16,7 & 33,3 \\
\hline Limitações Físicas & 0,96 & 15,7 & 22,5 & $0,0-100,0$ & 0,0 & 33,3 \\
\hline Limitações Sociais & 0,96 & 10,5 & 14,2 & $0,0-55,6$ & 0,0 & 22,2 \\
\hline Relações Pessoais & 0,80 & 10,5 & 19,0 & $0,0-66,7$ & 0,0 & 16,7 \\
\hline Emoções & 0,94 & 15,6 & 27,2 & $0,0-88,9$ & 0,0 & 11,1 \\
\hline Sono e Disposição & 0,55 & 7,1 & 12,3 & $0,0-33,3$ & 0,0 & 16,7 \\
\hline Medidas de Gravidade & 0,78 & 28,3 & 23,9 & $0,0-100,0$ & 16,7 & 29,2 \\
\hline
\end{tabular}

Fonte: Dados da pesquisa, 2012. 


\section{Qualidade de vida relacionada à saúde no pós-parto}

As pontuações alcançadas nos diferentes domínios do KHQ durante o seguimento são apresentadas nas Tabelas 3 e 4. No terceiro mês após o parto, Tabela 3, observa-se a análise de medidas de tendência central dos domínios do KHQ, que mostra maior média nos domínios: percepção geral de saúde $(35,7)$, medidas de gravidade $(28,3)$, impacto da incontinência $(25,7)$ e limitações das atividades diárias $(22,9)$.

Na Etapa 3, vista à Tabela 4, a média elevada ocorreu nos domínios: impacto da incontinência $(54,2)$, medidas de gravidade $(48,3)$, percepção geral da saúde $(46,3)$, limitações de atividades diárias $(34,6)$ e limitações físicas $(33,3)$. Estas médias elevadas em domínios de ambas as fases do estudo indicam os piores níveis de QV.

Tabela 4 - Análise de confiabilidade, escores médios, desvio-padrão, mediana, valores mínimos e máximos, para os domínios do KHQ aplicado às mulheres autorreferidas incontinentes - Etapa $3(n=40)$. Londrina, PR $-2012$.

\begin{tabular}{lcccccc}
\hline \multicolumn{1}{c}{ Domínios do KHQ } & $\begin{array}{c}\text { Alfa de } \\
\text { Cronbach }\end{array}$ & Média & $\begin{array}{c}\text { Desvio } \\
\text { Padrão }\end{array}$ & $\begin{array}{c}\text { Mínimo- } \\
\text { Máximo }\end{array}$ & $\begin{array}{c}\text { Mediana } \\
\text { IQR } \\
\text { (q3-q1) }\end{array}$ \\
\hline Percepção geral da Saúde & -- & 46,3 & 18,4 & $0,0-100,0$ & 50,0 & 25,0 \\
\hline Impacto da Incontinência & -- & 54,2 & 24,7 & $0,0-100,0$ & 66,7 & 33,3 \\
\hline Limitações de Atividades Diárias & 0,99 & 34,6 & 28,3 & $0,0-100,0$ & 33,3 & 20,8 \\
\hline Limitações Físicas & 0,95 & 33,3 & 24,5 & $0,0-100,0$ & 33,3 & 16,7 \\
\hline Limitações Sociais & 0,95 & 19,7 & 19,6 & $0,0-88,9$ & 11,1 & 11,1 \\
\hline Relações Pessoais & 0,82 & 17,1 & 22,2 & $0,0-66,7$ & 0,0 & 33,3 \\
\hline Emoções & 0,90 & 25,0 & 26,1 & $0,0-77,8$ & 11,1 & 47,2 \\
\hline Sono e Disposição & 0,90 & 30,8 & 23,7 & $0,0-100,0$ & 33,3 & 16,7 \\
\hline Medidas de Gravidade & 0,77 & 48,3 & 23,1 & $8,3-100,0$ & 50,0 & 35,4 \\
\hline
\end{tabular}

Fonte: Dados da pesquisa, 2012.

A seguir são apresentados os escores médios alcançados nos domínios do KHQ, de acordo com o tipo de IU autorreferida. Observa-se à Tabela 5, que na Etapa 2, mulheres autorreferidas com IU de urgência obtiveram pontuações médias significativamente mais elevadas, exceto nos domínios impacto da incontinência e sono e disposição.

Tabela 5 - Associação da média (dp) dos valores dos escores obtidos para cada domínio do KHQ de acordo com o tipo de IU no pós-parto - Etapa 2 ( $n=35)$. Londrina, 2012.

\begin{tabular}{lccccc}
\hline & IU de esforço & IU de urgência & IU Mista & \\
\cline { 2 - 4 } \multicolumn{1}{c}{ Domínios do KHQ } & Média (dp) & Média (dp) & Média (dp) & p-valor \\
\hline Percepção geral da Saúde & $31,8(26,9)$ & $50,0(20,4)$ & $38,9(13,2)$ & 0.1115 \\
Impacto da Incontinência & $27,3(33,5)$ & $25,0(50,0)$ & $22,2(23,6)$ & 0.804 \\
Limitações de Atividades Diárias & $22,7(31,1)$ & $33,3(47,1)$ & $18,5(17,6)$ & 0.945 \\
Limitações Físicas & $12,9(23,5)$ & $20,8(31,5)$ & $20,4(16,2)$ & 0.297 \\
Limitações Sociais & $8,6(14,5)$ & $16,7(21,3)$ & $12,3(10,3)$ & 0.3901 \\
Relações Pessoais & $9,8(18,3)$ & $16,7(33,3)$ & $9,3(14,7)$ & 0.9772 \\
Emoções & $15,2(28,4)$ & $25,0(42,9)$ & $12,3(17,1)$ & 0.9001 \\
Sono e Disposição & $9,8(14,2)$ & $4,2(8,3)$ & $1,9(5,6)$ & 0.2942 \\
\hline Medidas de Gravidade & $25,8(22,6)$ & $35,4(43,2)$ & $31,5(18,5)$ & 0.5381 \\
\hline Fonte: Dados da pesquisa, 2012. & & Valor p calculado pelo teste Kruskal-Wallis.
\end{tabular}


Na Tabela 6, com dados da Etapa 3, observa-se que, com exceção dos domínios percepção geral da saúde e sono e disposição, as pontuações médias mais elevadas estão entre as mulheres com IU de esforço.

Tabela 6 - Associação da média (dp) dos valores dos escores obtidos para cada domínio do KHQ de acordo com o tipo de IU no pós-parto - Etapa 3 ( $n=40)$. Londrina, 2012.

\begin{tabular}{lccccc}
\hline \multirow{1}{*}{ Domínios do KHQ } & IU de esforço & IU de urgência & \multicolumn{1}{c}{ IU Mista } & p-valor \\
\cline { 2 - 5 } & Média (dp) & Média (dp) & Média (dp) & \\
\hline Percepção geral da Saúde & $45,8(20,4)$ & $41,7(14,4)$ & $48,1(16,0)$ & 0.8121 \\
Impacto da Incontinência & $55,6(25,4)$ & $44,4(19,2)$ & $53,8(25,6)$ & 0.7168 \\
Limitações de Atividades Diárias & $37,5(26,6)$ & $11,1(19,2)$ & $34,6(32,2)$ & 0.2436 \\
Limitações Físicas & $34,7(26,9)$ & $27,8(9,6)$ & $32,1(23,0)$ & 0.918 \\
Limitações Sociais & $21,8(22,8)$ & $11,1(0,0)$ & $17,9(14,7)$ & 0.6753 \\
Relações Pessoais & $18,8(22,7)$ & $0,0(0,0)$ & $17,9(23,0)$ & 0.2758 \\
Emoções & $28,2(28,2)$ & $3,7(6,4)$ & $23,9(23,5)$ & 0.2081 \\
Sono e Disposição & $31,9(26,9)$ & $16,7(16,7)$ & $32,1(18,6)$ & 0.5123 \\
\hline Medidas de Gravidade & $51,4(22,1)$ & $27,8(9,6)$ & $47,4(25,5)$ & 0.2301 \\
\hline Fonte: Dados da pesquisa, 2012. & & Valor p calculado pelo teste Kruskal-Wallis.
\end{tabular}

De acordo com a proposta do KHQ, as médias mais elevadas observadas às Tabelas 5 e 6 demonstram os domínios que mais prejudicam a QVRS das mulheres incontinentes no PP, segundo o tipo de perda urinária. Os dados mostram ainda que durante o seguimento, não houve significância estatística entre os domínios do KHQ e os tipos de IU.

\section{DISCUSSÃO}

Os dados relacionados a incidência da IU e da QV do presente estudo fazem parte da segunda e terceira etapa de uma coorte com mulheres no PP. O seguimento das puérperas foi realizado nas primeiras 48 horas, no terceiro e sexto após o parto.

Estudos encontrados a luz da literatura, mostram que a experiência com episódios de perda urinária é uma condição que não prevalece somente em mulheres idosas, mas, também, em mulheres jovens e na meiaidade (LEROY LS e LOPES MHBM, 2012, MENEZES GMD, et al., 2012 e SABOIA DM, et al., 2017). A incidência de IU no terceiro mês após o parto de $10,5 \%$ e de $12,3 \%$ no sexto mês após o parto neste estudo está semelhante quando comparada àquelas encontradas em outros estudos (SOLANS-DOMENECH M, et al., 2010).

A IU acarreta efeitos e interferências negativas na vida em geral, seja na realização de rotinas diárias como também nas atividades de lazer e nos relacionamentos pessoal e social (DEDICAÇÃO AC, et al, 2009, MENEZES GMD, et al., 2012 e SABOIA DM, et al., 2017). Os aspectos de funcionamento físico, social e emocional, são significativamente prejudicados pela IU e a influência dos sinto, mas sobre a percepção da QVRS pode variar de acordo com a gravidade dos sintomas e o comprometimento relatados pelas entrevistadas (LASSERRE A, et al., 2009). Esses dados corroboram os achados da pesquisa conduzida na Austrália (O'REILLY R, et al., 2008) com 10 mulheres, das quais $20 \%$ referiram IU no PP, assim como a necessidade de usar absorvente continuamente, o que interferia em suas atividades diárias, na escolha do que vestir e nas atividades sociais. Outro estudo, realizado nos Estados Unidos, com 759 primíparas, cujo objetivo foi investigar o impacto de incontinências urinária e fecal no PP sobre a QV de mulheres, mostrou que seis meses após o parto as incontinências urinária e fecal têm importante efeito negativo na saúde das mulheres com impacto na sua QV (HANDA VL, et al., 2007).

Estudo realizado na China, mostrou que a IU causa efeitos negativos sobre a QVRS e que estes efeitos são maiores quando comparados com outras doenças crônicas, no entanto, são muitas vezes negligenciados 
(HORNG SS, et al., 2013). Autores relatam algumas dificuldades para comparar os dados encontrados na literatura sobre a QVRS, tais como as diferenças metodológicas e a variedade de questionários utilizados. Enfatizam que a IU pode afetar alguns domínios da QV, com ênfase nos domínios que dizem respeito à saúde física, mental, sexual e social das mulheres (FERNANDES S, et al., 2015, MENEZES GMD, et al., 2012 e SABOIA DM, et al., 2017).

Sabe-se que nos últimos anos, o interesse em avaliar a QVRS em pesquisas clínicas têm aumentado, e que existem vários questionários para se avaliar a QV. Com objetivo de traduzir e adaptar para o português o questionário de QV KHQ (TAMANINI JTN, et al., 2003), foi realizado estudo com 154 mulheres brasileiras com IU. Os resultados mostraram que o coeficiente alfa de Cronbach padronizado foi de 0,87 , a consistência interna dos domínios em separado, variou de 0,49 (sono/disposição) a 0,92 (emoções). Os achados mostraram ainda pontuação média elevada nos domínios impacto da incontinência, emoções, medidas de gravidade, limitações de atividades diárias, e limitações físicas. Os autores concluíram que o KHQ representa um importante instrumento para a avaliação de mulheres incontinentes em pesquisa clínica e que a versão para o idioma português do instrumento mostrou-se confiável e válida. A aplicação do $\mathrm{KHQ}$ em estudo posterior, mostrou alta confiabilidade e validade, confirmando sua viabilização para estudos brasileiros sobre IU (FONSECA ESM, et al., 2005).

Diante dessa situação, vislumbrou-se a necessidade de realizar investigação sobre a QVRS das mulheres incontinentes e o instrumento utilizado neste estudo, teve a finalidade de demonstrar o quanto a perda de urina afeta a QVRS das mulheres incontinentes no terceiro e no sexto mês após o parto. A consistência interna do KHQ, medida pelo coeficiente Alfa de Cronbach padronizado, foi satisfatória, obtendo-se índice geral de 0,93 na Etapa 2 e de 0,96 na Etapa 3, excedendo o valor mínimo geralmente utilizado como referência de 0,70 .

Recorde-se que a aplicação do alfa de Cronbach para cada domínio do KHQ em separado, na Etapa 2, mostrou entre 0,55 (sono/disposição) a 0,98 (limitações das atividades diárias), que indicam a consistência interna do instrumento variando entre regular e excelente. Na Etapa 3, a consistência interna dos domínios variou de 0,77 (medidas de gravidade) a 0,99 (limitações das atividades diárias), que indicam níveis entre bom e excelente em sua consistência interna.

Ao avaliar a QVRS de puérperas incontinentes por meio do KHQ, observou-se no terceiro mês PP médias maiores nos domínios percepção geral de saúde, medidas de gravidade, impacto da incontinência e limitações das atividades diárias, e no sexto mês as médias elevadas foram nos domínios impacto da incontinência, medidas de gravidade, percepção geral da saúde, limitações de atividades diárias e limitações físicas, indicando os piores níveis de qualidade de vida relacionada à saúde das puérperas incontinentes. Diante destes dados, pode-se supor que a QVRS é mais afetada no sexto mês PP.

Os resultados aqui, não diferem de outros estudos, que utilizaram o mesmo instrumento para avaliar a QVRS, e mostraram médias mais altas nos domínios medida de gravidade, percepção geral de saúde e impacto da incontinência (LIMA JLDA, 2009), impacto da incontinência, emoções, limitações das atividades diárias e limitações físicas ( DEDICAÇÃO AC, et al., 2009, HANDA VL, et al., 2007 e LEROY LS e LOPES MHBM, 2012).

Em relação ao tipo de IU, IU Mista foi a mais prevalente em alguns estudos (DEDICAÇÃO AC, et al., 2009, LEROY LS e LOPES MHBM, 2012, RIESCO MLG et al., 2014 e TAMANINI JTN, et al., 2003). Isso indica que a IUM é responsável por maior impacto na QVRS, quando comparada com a IU de esforço e de urgência, e revela a importância de se implementar tratamento específico para esse tipo de IU. Os resultados aqui, diferem destes estudos, pois notou-se que na Etapa 2 a QVRS foi considerada pior quando o tipo de queixa era IU de urgência em todos os domínios do $\mathrm{KHQ}$, com exceção dos domínios impacto da incontinência e sono/disposição, enquanto na Etapa 3 o tipo de incontinência que mais afetou a QVRS das mulheres foi a IU de esforço, com exceção dos domínios percepção geral de saúde e sono/disposição.

Sob o ponto de vista da QVRS, cabe esclarecer que há uma carência de estudos atuais com esta temática e que para comparar os resultados de diferentes estudos, optou-se por aqueles que empregaram o mesmo 
questionário e cuja população fosse semelhante, de modo a garantir resultados mais consistentes e satisfatórios. Os achados demonstraram que a IU afetou de forma significativa a QVRS, porém, diante da escassez de investigações sobre esta temática, observa-se a necessidade de mais estudos que empreguem o KHQ na investigação da QVRS de puérperas incontinentes.

Merece destaque também o fato de que, no decorrer das etapas desta pesquisa, tornou-se evidente a carência de informações e/ou conhecimento sobre IU das mulheres. Diante deste fato, verificou-se a necessidade de oferecer às entrevistadas, informações e/ou esclarecimentos sobre IU e, esclarecer medidas que ajudam a prevenir a ocorrência de IU. Cabe esclarecer ainda, que foi utilizado um roteiro para guiar as orientações oferecidas às mulheres e, que o mesmo foi utilizado em pesquisa anterior realizada em São Paulo (LOPES DBM e PRAÇA NS, 2010). O conteúdo deste roteiro abordava os seguintes temas: evitar reter a urina; criar o hábito de urinar com intervalo de poucas horas; manter peso corporal saudável; evitar constipação intestinal; buscar tratamento para qualquer tosse persistente; evitar levantar muito peso; realizar exercício para fortalecer o músculo do assoalho pélvico; comunicar o profissional de saúde (médico ou enfermeira) se apresentar perda de urina e não deixar de ingerir líquido.

Embora não coloque diretamente em risco a vida, a IU pode trazer sérias implicações incapacitantes e significativa morbidade entre as mulheres no período PP. Ainda que não se apresente como situação associada, chama atenção o comportamento da mulher incontinente não relatar o agravo ao profissional de saúde. O elevado percentual de mulheres nessa situação demonstra uma demanda reprimida por assistência diante desse agravo no PP.

Diante dos resultados apresentados, observa-se a necessidade de mais estudos que investiguem a QVRS de puérperas incontinentes através do $\mathrm{KHQ}$, e considera-se fundamental que o enfermeiro esteja atento a esta problemática e consiga intervir precocemente a fim de ajudar a mulher a prevenir a IU, pois conhecer os domínios da QVRS afetados pela IU é de fundamental importância para que se possa atuar de forma mais direcionada, a fim de contribuir para melhoria da saúde e bem-estar dessa população.

Como limitação do estudo pode-se apontar a falta de padronização dos instrumentos utilizados para avalias a QRVS de mulheres com IU.

\section{CONCLUSÃO}

Esta pesquisa mostrou incidência de IU autorreferida no pós-parto e está morbidade causa impacto negativo na QVRS das puérperas incontinentes. Utilizando-se o KHQ, observou-se impacto elevado da IU nos domínios a percepção geral de saúde, medidas de gravidade, impacto da incontinência, limitações das atividades diárias e limitações físicas, e que a QVRS está mais prejudicada no sexto mês pós-parto quando se compara ao terceiro mês precedente, uma vez que é naquele momento que se observam as maiores médias dos domínios do KHQ. O estudo concluiu que no terceiro mês após o parto, a IU de urgência foi a mais prevalente, enquanto no sexto mês houve prevalência maior foi de IU de esforço. Quanto à avaliação da QVRS, independentemente da classificação recebida pela mulher incontinente, todas tiveram sua qualidade de vida afetada negativamente. Os objetivos dessa pesquisa foram alcançados à medida que se conseguiu revelar a incidência e o impacto que a IU acarreta na QV das puérperas, por meio dos domínios do KHQ. Sugere-se que por meio deste estudo, novas pesquisas sejam realizadas e que os profissionais de enfermagem sejam sensibilizados para o direcionamento da problemática e para a construção do conhecimento na área da saúde da mulher, no intuito de prevenir e tratar a IU, garantindo dessa forma a QVRS das mulheres incontinentes.

\section{REFERÊNCIAS}

1. ABRAMS P, et al. ICl Book. 3 rd International Consultation on Incontinence - recommendations Committee: Evaluation and Treatment of Urinary Incontinence, Pelvic Organ Prolapse and Fecal Incontinence. 2005; 26-29.

2. DEDICAÇÃO AC, et al. Comparação da qualidade de vida nos diferentes tipos de incontinência urinária feminina. Rev. bras. fisioter. 2009; 13(2): 116-22. 
3. FARIA CA, et al. Impacto do tipo de incontinência urinária sobre a qualidade de vida de usuárias do Sistema Único de Saúde no Sudeste do Brasil. Rev Bras Ginecol Obstet. 2015; 37(8):374-80.

4. FERNANDES S, et al. Qualidade de vida em mulheres com Incontinência Urinária. Revista de Enfermagem Referência. 2015; 5: 93-99. Disponível em: http://dx.doi.org/10.12707/RIV14042

5. FONSECA ESM, et al. Validação do questionário de qualidade de vida (King's Health Questionnaire) em mulheres brasileiras com incontinência urinária. Rev Bras Ginecol Obstet. 2005; 27(5): 235-42.

6. HANDA VL, et al. The impact of fecal and urinary incontinence on quality of life 6 months after childbirth. Am $\mathrm{J} O b s t e t$ Gynecol. 2007;197(6):636.e1-e6.

7. HORNG SS, et al. The epidemiology of Urinary Incontinence and it's Influence on Quality of Life in Taiwanese MiddleAged Women. Neurourology and Urodynamics. 2013; 32:371-76.

8. KELLEHER CJ, et al. A new questionnaire to assess the quality of life of urinary incontinent women. Br $\mathrm{J}$ Obstet Gynaecol 1997;104:1374-9.

9. LASSERRE A, et al. Urinary Incontinence in French women: Prevalence, risk factors, and impact on quality of life. Eur Urol. 2009; 56: 177-83.

10. LEROY LS, LOPES MHBM. A incontinência urinária no puerpério e o impacto na qualidade de vida relacionada à saúde. Rev. Latino-Am. Enfermagem. 2012; 20(2): 346-53.

11. Lima JLDA. Incontinência urinária no ciclo gravídico-puerperal e impacto na qualidade de vida. Dissertação (Mestrado em Enfermagem) - Faculdade de Ciências Médias, Universidade Estadual de Campinas, Campinas; 2009.

12. LOPES DBM, PRAÇA NS. Incontinência Urinária Autorreferida no Pós-Parto.Texto Contexto Enferm, Florianópolis, 2010 Out-Dez; 19(4): 667-74.

13. MENEZES GMD, et al. Queixa de perda urinária: um problema silente pelas mulheres. Rev Gaúcha Enferm., Porto Alegre (RS) 2012 mar;33(1):100-8.

14. O'REILLY R, et al. Women's experiences of recovery from childbirth: focus on pelvis problems that extend beyond the puerperium. J Clin Nurs. 2008; 18(14):2013-9.

15. RAZA-KHAN F, et al. Peripartum urinary incontinence in a racially diverse obstetrical population. Int Urogynecol J Pelvic Dysfunct. 2006; 17(5):525-30.

16. RIESCO MLG, et al. Incontinência urinária relacionada à força muscular perineal no primeiro trimestre da gestação: estudo transversal. Rev Esc Enferm USP 2014; 48(Esp):33-9

17. SABOIA DM, et al. Impacto dos tipos de incontinência urinária na qualidade de vida de mulheres. Rev Esc Enferm USP · 2017;51:e03266

18. SOLANS-DOMÈNECH M, et al. Urinary and Anal Incontinence During Pregnancy and Postpartum: Incidence, Severity, and Risk Factors. Obstet Gynecol. 2010; 115:618-28.

19. STREINER DI. Being inconsistent about consistency: when coefficient alpha does and doesn't matter. Journal of Personality Assessment. 2003; 80: 217-222.

20. TAMANINI JTN, et al. Validação do "King's Health Questionnaire" para o português em mulheres com incontinência urinária. Rev Saúde Pública. 2003;37(2):203-11.

21. WHOQOL. The World Health Organization Quality of Life assessment: position paper from the World Health Organization. special issue .quality of life. soc sci med. 1995; 41(10):1403-9. 